University of Nebraska - Lincoln

DigitalCommons@University of Nebraska - Lincoln

6-2003

\title{
A combined heating cooling stage for cluster thermalization in the gas phase
}

D. N. levlev

Max Planck Institute for Solid State Research

A. Küster

Max Planck Institute for Solid State Research

Axel Enders

University of Nebraska-Lincoln, a.enders@me.com

N. Malinowski

Max Planck Institute for Solid State Research

H. Schaber

Max Planck Institute for Solid State Research

See next page for additional authors

Follow this and additional works at: https://digitalcommons.unl.edu/physicsenders

Part of the Physics Commons

levlev, D. N.; Küster, A.; Enders, Axel; Malinowski, N.; Schaber, H.; and Kern, Klaus, "A combined heating cooling stage for cluster thermalization in the gas phase" (2003). Axel Enders Publications. 12.

https://digitalcommons.unl.edu/physicsenders/12

This Article is brought to you for free and open access by the Research Papers in Physics and Astronomy at DigitalCommons@University of Nebraska - Lincoln. It has been accepted for inclusion in Axel Enders Publications by an authorized administrator of DigitalCommons@University of Nebraska - Lincoln. 


\section{Authors}

D. N. levlev, A. Küster, Axel Enders, N. Malinowski, H. Schaber, and Klaus Kern 


\title{
A combined heating cooling stage for cluster thermalization in the gas phase
}

\author{
D. N. levlev, ${ }^{\text {a) }}$ A. Küster, A. Enders, N. Malinowski, ${ }^{\text {b) }}$ H. Schaber, and K. Kern \\ Max Planck Institute for Solid State Research, Heisenbergstrasse 1, 70569 Stuttgart, Germany
}

(Received 23 January 2003; accepted 12 March 2003)

\begin{abstract}
We report on the design and performance of a combined heating/cooling stage for the thermalization of clusters in a gas phase time-of-flight mass spectrometer. With this setup the cluster temperature can sensitively be adjusted within the range from 100 up to $800 \mathrm{~K}$ and higher. The unique combination of a heating stage with a subsequent cooling stage allows us to perform thermodynamic investigations on clusters at very high temperatures without quality losses in the spectra due to delayed fragmentation in the drift tube of the mass spectrometer. The performance of the setup is demonstrated by the example of $\left(\mathrm{C}_{60}\right)_{n}$ clusters. (C) 2003 American Institute of Physics.
\end{abstract}

[DOI: $10.1063 / 1.1573748$ ]

\section{INTRODUCTION}

Time-of-flight (TOF) mass spectrometry is a powerful technique for the exploration of the structure of small clusters in the gas phase. In such experiments information about the cluster structure can be deduced from the measurement of the thermal cluster stability as a function of the cluster size. Heating of a cluster causes evaporation of atoms or molecules, reducing the cluster size. Clusters of a particularly stable structure stronger resist this evaporation process and can be identified by a set of pronounced peaks in a mass spectrum taken at elevated temperatures. ${ }^{1-5}$ Moreover experiments probing thermodynamic properties require the cluster temperature to be varied in order to melt or thermalize clusters. For instance, well-defined cluster temperatures are necessary for the determination of the heat capacity and melting point of free sodium clusters, ${ }^{6-8}$ the activation energies and evaporation rate for the evaporation of weakly bound sodium clusters, ${ }^{9,10}$ as well as the study of the decay behavior of fullerene dimers. ${ }^{11,12}$

Mainly two approaches are commonly used to vary the cluster temperature experimentally. On the one hand, the cluster can be heated by the absorption of laser light. ${ }^{1-3,13}$ In this case, several photons of the laser light illuminating the clusters en route are absorbed within a few nanoseconds. The final cluster temperature depends on many parameters, such as the photon energy, laser power, and absorption cross section, and is usually not known. Nevertheless, very high temperatures sufficient to completely fragment even the most stable clusters can be achieved in this case. On the other hand, thermalization of the cluster within a noble gas bath allows us to precisely adjust the cluster temperature. Here, after leaving the source the clusters and the helium transport gas pass a heating stage of a certain temperature within milliseconds. The temperature of the stage is mediated to the

\footnotetext{
a) Author to whom correspondence should be addressed; electronic mail: d.ievlev@fkf.mpg.de

b) Permanent address: Central Laboratory of Photoprocesses, Bulgarian Academy of Sciences, 1040 Sofia, Bulgaria.
}

clusters by collisions with the He atoms. The main advantage of the collision induced thermalization compared to the absorption technique is that it allows adjusting well-defined cluster temperatures within a very broad temperature range.

In this article we report on the design and the performance of a combined heating and cooling stage. The setup allows thermalizing the cluster beam to well-defined temperatures between 100 and $800 \mathrm{~K}$. The innovative feature of the setup is the possibility to decrease the cluster temperature rapidly by a separate cooling stage after passing the heater. Thus, the cluster structure formed within the heating stage is quenched and further evaporation of atoms is drastically reduced. Undesired delayed cluster fragmentation within the drift tube of the mass spectrometer, which decreases resolution or even completely prevents well-resolved peaks, is suppressed. The temperature profile of the He transport gas is measured by a thermocouple, which was moved along the cluster beam direction inside the stage. The benefit of the setup for the investigation of thermally induced structural changes in clusters of $\mathrm{C}_{60}$ molecules is demonstrated.

\section{EXPERIMENTAL SETUP}

The design of the combined heating and cooling stage is schematically depicted in Fig. 1. The heating cell is a further development of the heating nozzle used for the study of size-dependent melting of sodium clusters. ${ }^{14}$ The new design was inspired by similar cells used by other experimentalists. ${ }^{6,9,15,16}$ The main part is a $120 \mathrm{~mm}$ long copper tube (1) of $6 \mathrm{~mm}$ inner diameter, which is attached directly to the exit nozzle of the cluster source (2). Thus, the clusters enter the heating stage immediately after leaving the source. The tube is thermally isolated from the condensation cell by a Teflon washer (3). It is heated resistively by a coaxial heating wire (4) wound around it with changing pitch. The assembly is wrapped by a dense copper wire netting (5), which is thermally connected to liquid nitrogen cooling of the condensation cell. Finally, the heating stage is mantled by a stainless steel shield (6). Due to the cold copper wire netting temperatures below room temperature can also be 
Thermocouple position, $\mathrm{cm}$

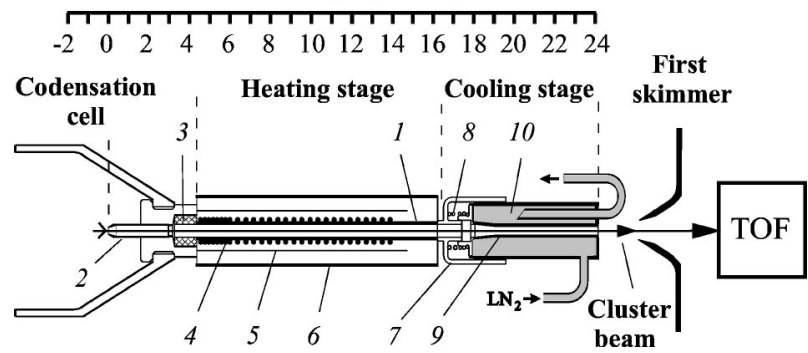

FIG. 1. Scheme of the experimental setup. After leaving the condensation cell the cluster successively pass the heating and the cooling stage. The scale on top refers to the thermocouple position during temperature profile measurements (see Fig. 2).

achieved when the heating current is only small or zero. Thus, with the heating cell the cluster temperature can be sensitively adjusted within the range from 100 to $800 \mathrm{~K}$.

We find that the cluster intensity in the TOF spectra decreases rapidly with increasing tube length. Therefore, the length of the tube was optimized in order to achieve a constant temperature profile, i.e., long enough to thermalize the clusters while still maintaining sufficient beam intensity. Particular care has been taken to minimize the temperature variation within the heating tube at all temperatures (see Fig. 2). This was accomplished by winding the heating wire with a pitch that changes with position. At the entrance of the heating tube eight turns with a high pitch of about $2 \mathrm{~mm}$ per turn are wound, followed by 23 turns of lower pitch of about $4 \mathrm{~mm}$ per turn until the exit of the tube. Thus, when operating at elevated temperatures the cold clusters are heated up quickly and the hot zone of the tube is maximized.

The hot clusters enter the cooling stage directly after leaving the heater. Both stages are mechanically connected by a quartz glass adapter (7) and a spring (8). This flexible connection avoids intensity losses during the transfer of the clusters while keeping the heat conduction at a minimum. The central part of the cooling stage is a stainless steel tube (9) with inner diameter that reduces from 6 to $4 \mathrm{~mm}$ within a distance of $7 \mathrm{~mm}$ at the entrance of the tube. The tube is embedded inside a flow cryostat with a reservoir of $\sim 3 \mathrm{~cm}^{3}$ for $\mathrm{LN}_{2}$ or $\mathrm{LHe}$ coolants. For the applications shown in this

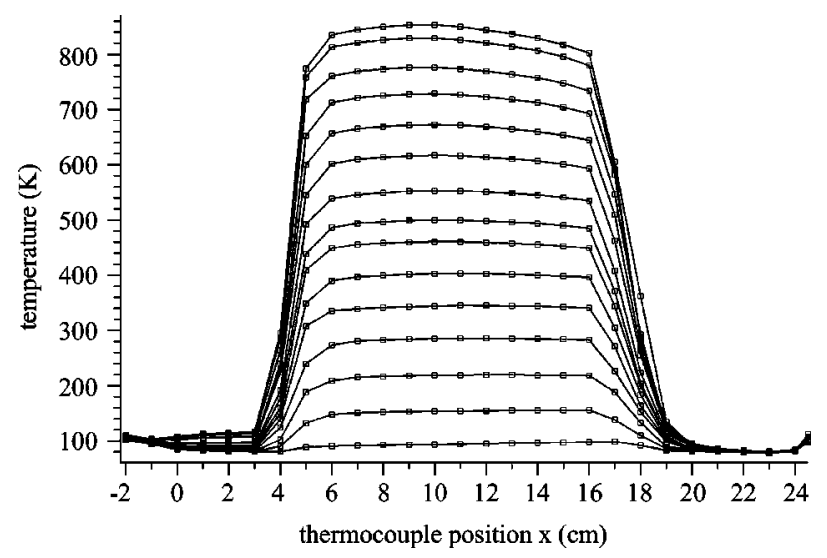

FIG. 2. Temperature profile of the He transport gas inside the heating/ cooling stage. The temperature was measured by a chromel-constantan thermocouple. article the cryostat was operated with liquid nitrogen.

Due to the pressure gradient between the cluster source and the first skimmer the pressure is already significantly decreased inside the cooling stage, reducing the number of collisions of the cluster with the He bath and increasing the tube length necessary for cluster thermalization. The purpose of the reduced inner diameter of the cold tube is to increase the cooling efficiency by increasing the collision rate. The attempt to increase the He partial pressure inside the cold tube and thus the cooling speed by injecting cold $\mathrm{He}$ gas at the circumference of the cooling stage entrance resulted in a complete loss of the cluster signal and was not successful.

The assembly is placed within our reflectron TOF mass spectrometer, ${ }^{11}$ between the low-pressure inert gas condensation cell ${ }^{17}$ and the first skimmer (Fig. 1). The TOF allows high-resolution mass spectroscopy with a resolution of $\mathrm{m} / \mathrm{\Delta m}$ $=15.000$ within the mass range up to $20.000000 \mathrm{amu}$. The clusters are transported within a He flow driven by the pressure gradient from several millibar inside the cluster source down to $10^{-7} \mathrm{mbar}$ in the drift tube. The cluster velocity, and thus the dwell time of the clusters within the heating stage, has been determined experimentally. ${ }^{11,12}$ This was done by measuring the different flight time of clusters by alternatively ionizing the clusters at the entrance and the exit of the heating stage. The dwell time can be varied from 0.5 to $1 \mathrm{~ms}$ by changing the helium pressure inside the cluster source.

\section{PERFORMANCE OF THE SETUP}

\section{A. Temperature profile inside the combined heating/cooling stage}

The temperature inside the heating/cooling stage as a function of position and heating power was measured by using a chromel-constantan thermocouple. During the measurements the thermocouple was moved stepwise along the cluster path, starting from inside the cluster source until the exit of the cooling cell. Before acquiring the temperature at every position the thermocouple has completely been thermalized with the He transport gas. This was recognized by plateaus in the time dependence of the thermovoltage. The obtained temperature profile for different heating powers is shown in Fig. 2. The data represent the temperature of the $\mathrm{He}$ transport gas. During the measurements the cooling stage and the cluster source have been cooled by liquid nitrogen. The position on the $x$ axis in Fig. 2 corresponds to the position indicated in Fig. 1. The thermocouple was attached electrically isolated to the system. Any mechanical contact with the heating stage, identified by a drop in the electrical resistance to ground, could thus be avoided.

According to Fig. 2 the cluster temperature profile can be characterized as follows: (i) the temperature inside the heating zone is constant and (ii) the temperature inside the cluster source is affected only little by the heating. On the other hand, the thermal coupling between heating and cooling stage is moderate and shows up only at high temperatures above $600 \mathrm{~K}$. (iii) The transition regions between plateaus of constant low and high temperatures are extremely short, which on the other hand extends the plateau length. The cold helium emerging from the source is heated up to 
$840 \mathrm{~K}$ within only $3 \mathrm{~cm}$. On the other hand, the cooling stage reduces the helium temperature from $840 \mathrm{~K}$ down to $<100 \mathrm{~K}$ within $4 \mathrm{~cm}$.

For a thermalization of the clusters at well-defined temperatures the constancy of the temperature inside the heating zone is very crucial. Plateaus of constant temperature are found over a length of $10 \mathrm{~cm}$. The deviation from the average plateau temperature increases with increasing heating power: Below $600 \mathrm{~K}$ the plateau temperature is constant within $\pm 5 \mathrm{~K}$ over $10 \mathrm{~cm}$, while at higher temperatures the deviation is smaller than $\pm 15 \mathrm{~K}$. Deviations occur mainly at the ends of the heating zone due to heat transfer to the adjacent cold zones. Much attention was paid to the optimization of the plateau by modifying the pitch of the heating wire, in particular at the ends of the heating stage. Experiments with separate heating coils at the ends to compensate losses gave only little improvement, but required significantly higher efforts to control the He temperature during the experiment.

The He transport gas represents the temperature of the clusters only if the clusters perform a sufficient number of collisions with the He atoms. This number varies with $\mathrm{He}$ pressure, the collision cross section, the average cluster velocity with respect to the helium atoms, as well as the time the clusters spend inside the heating zone. We calculated that $\left(\mathrm{C}_{60}\right)_{n}$ clusters perform $10^{5}-10^{6}$ collisions with the He bath at a pressure of 1 mbar inside the cluster source, with the heating stage kept at $300 \mathrm{~K}^{11}$ The calculation shows that even at the highest temperature the length of the heating cell is sufficient for the clusters to perform enough collisions and to thermalize to the temperature given by the He bath.

In contrast to that, the cluster temperature inside the cooling stage is not in general represented by the He transport gas temperature. This is due to the reduced He partial pressure there and the reduced length of the cooling stage. The data in Fig. 2 show that the He has a temperature of 100 $\mathrm{K}$ over a distance of $4 \mathrm{~cm}$, from $x=20$ to $x=24$. However, the $\left(\mathrm{C}_{60}\right)_{n}$ clusters do not cool down to the bath temperature if the heating stage is at very high temperatures above $800 \mathrm{~K}$. This can be shown by means of delayed fragmentation in the mass spectra, as will be discussed in the next section.

\section{B. Temperature dependent TOF mass spectra}

The advantage of the combined heating/cooling stage for structural investigations is demonstrated by the example of $\left(\mathrm{C}_{60}\right)_{n}$ clusters. Figure 3 shows the mass spectra of $\left(\mathrm{C}_{60}\right)_{n}$ clusters taken at different cluster temperatures. For mass spectroscopy, the clusters have been ionized in the acceleration region of the TOF by a $20 \mathrm{~ns}$ light pulse of a $F_{2}$ excimer laser. The wavelength of the laser of $\lambda=157 \mathrm{~nm}$ corresponds to a photon energy of $7.9 \mathrm{eV}$. This energy is just above the $\mathrm{C}_{60}$ molecule ionization threshold $(7.6 \mathrm{eV})$, therefore clusters are ionized without additional heating.

The spectrum in Fig. 3(a) was obtained without any heating or cooling of the clusters after leaving the condensation cell. In this case, due to the thermal coupling of the heating stage to the condensation cell, the temperature of the cluster beam is $\sim 100 \mathrm{~K}$ after passing the heating/cooling stage. The appearance of well resolved peaks with a smooth

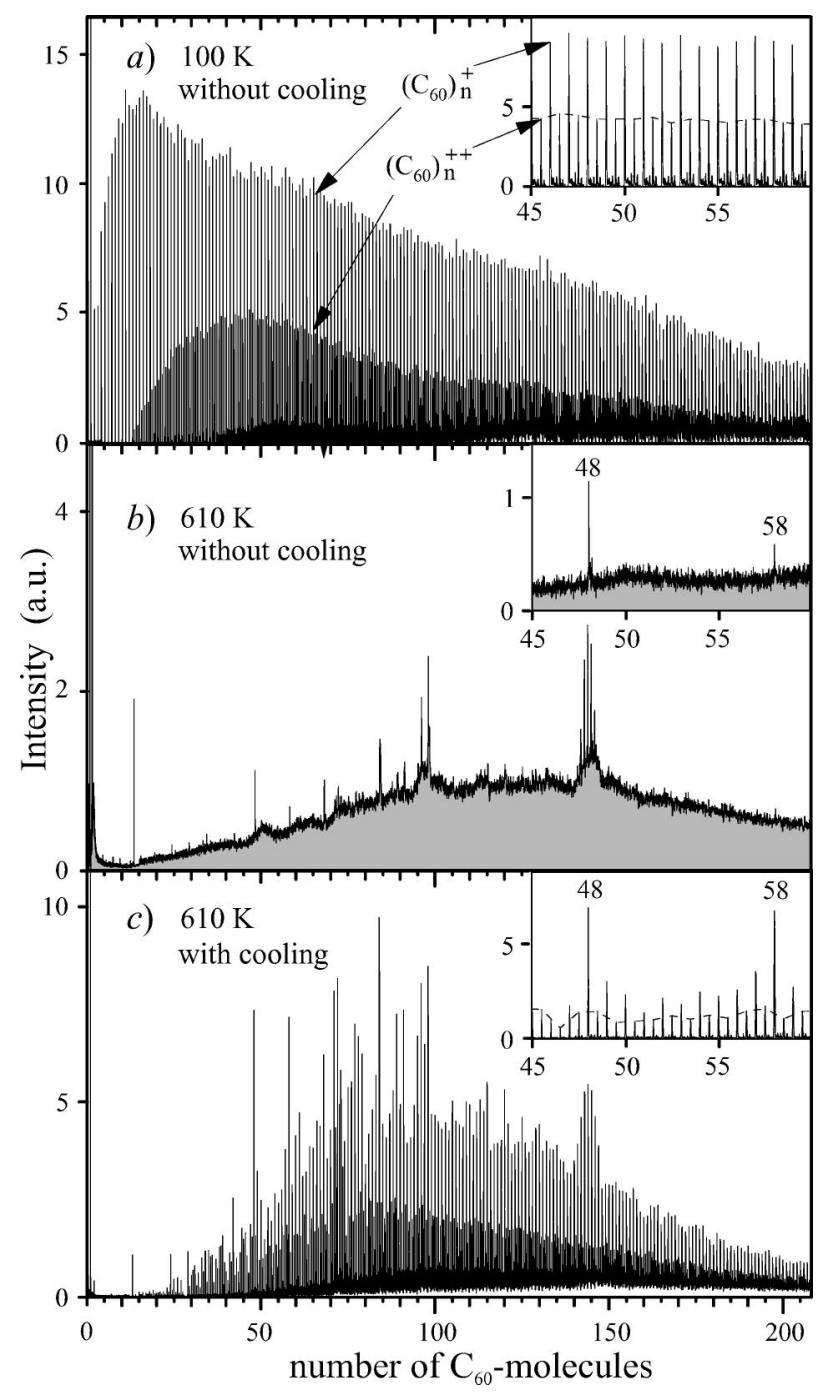

FIG. 3. Comparison of the TOF mass spectra of $\left(\mathrm{C}_{60}\right)_{n}$ clusters obtained (a) without thermal treatment, (b) after annealing the cluster beam to $610 \mathrm{~K}$, and (c) after annealing the clusters to $610 \mathrm{~K}$, followed by cooling with liquid nitrogen. The regions around $n=50$ are shown enlarged as insets in (a)-(c). Well-resolved peaks of doubly charged $\left(\mathrm{C}_{60}\right)_{n}^{++}$clusters, connected by dashed lines, are visible in the insets in (a) and (c). The background shaded in gray in (b) is due to delayed fragmentation in the drift tube of the mass spectrometer and cannot be resolved. The suppression of the delayed fragmentation leads to significant improvements of the spectra in (c).

intensity distribution, i.e., without particularly pronounced peaks, is characteristic for the disordered state of the $\left(\mathrm{C}_{60}\right)_{n}$ clusters. In addition to peaks of singly charged clusters $\left(\mathrm{C}_{60}\right)_{n}^{+}$, also the signal from doubly charged clusters $\left(\mathrm{C}_{60}\right)_{n}^{++}$is visible here [see the inset to Fig. 3(a)].

Heating the cluster beam up to $T=610 \mathrm{~K}$ without subsequent cooling completely changes the spectrum [Fig. 3(b)]: only a few prominent peaks on top of a nonresolved background, shaded in gray, are visible. These peaks represent particularly stable clusters, which resist evaporation during the time of flight. This clearly shows the main advantage of measurements at elevated temperatures for structural investigations. Less stable clusters sublime and their respective peaks disappear from the spectrum. The background shaded in gray is due to continuing evaporation of the hot clusters after leaving the heating cell, in the drift tube of the TOF. 
Such a delayed fragmentation inhibits or even completely prevents mass spectroscopy at elevated temperatures.

The delayed fragmentation can be completely suppressed by the cooling stage attached directly to the exit of the heating stage. After passing the hot zone the cluster temperature is quickly quenched below a value where further cluster fragmentation does not occur within the flight time to the mass detector. The mass spectrum in Fig. 3(c), taken at the same heating cell temperature as in Fig. 3(b) but with the cooling stage operated with liquid nitrogen, clearly reflects the improvement. Despite the high temperature, now clearly resolved peaks are visible. These peaks represent "magic" numbers of clusters of particularly stable configurations, from which the cluster structure can be determined. Here, cluster evaporation is confined exclusively to the heating stage. Any observed transformation of the cluster structure can be correlated with the temperature and the dwell time inside the heating stage. The set of magic numbers visible in this spectra corresponds to close-packed structures, decahedral structures, and structures based on the 98-Leary tetrahedron. ${ }^{18}$ The use of the heating stage for the detailed investigation of the so-called thermally induced structural transformation of $\left(\mathrm{C}_{60}\right)_{n}$ clusters is described in Refs. 12 and 19.

However, we have reason to believe that in some cases the cluster temperature after leaving the cooling stage is higher than the measured He temperature. We still find noticeable delayed fragmentation in the spectra when operating at high heating stage temperatures, above $800 \mathrm{~K}$. This means that the cluster temperature must be significantly higher than the $\mathrm{He}$ gas of $100 \mathrm{~K}$. The incomplete thermalization is due to the short length of the cooling stage and the low He pressure inside. Improvements will be achieved by extending the length of the cooling stage. Nevertheless, even at such high temperatures the cooling stage significantly reduces delayed fragmentation and essentially improve the mass spectra.

${ }^{1}$ C. Bréchignac, Ph. Cahuzac, J. Leygnier, and J. Weiner, J. Chem. Phys. 90, 1492 (1989)

${ }^{2}$ T. P. Martin, S. Bjørnholm, J. Borggreen, C. Bréchignac, Ph. Cahuzac, K. Hansen, and J. Pedersen, Chem. Phys. Lett. 186, 53 (1991).

${ }^{3}$ C. Bréchignac, H. Busch, Ph. Cahuzac, and J. Leygnier, J. Chem. Phys. 101, 6992 (1994).

${ }^{4} \mathrm{C}$. Bréchignac, in Clusters of Atoms and Molecules I, edited by H. Haberland (Springer, Berlin, 1994), p. 255.

${ }^{5}$ T. P. Martin, Phys. Rep. 273, 199 (1996).

${ }^{6}$ M. Schmidt, R. Kusche, W. Kronmüller, B. von Issendorff, and H. Haberland, Phys. Rev. Lett. 79, 99 (1997).

${ }^{7}$ R. Kusche, Th. Hippler, M. Schmidt, B. von Issendorff, and H. Haberland, Eur. Phys. J. D 9, 1 (1999).

${ }^{8}$ M. Schmidt, R. Kusche, T. Hippler, J. Donges, W. Kronmuller, B. von Issendorff, and H. Haberland, Phys. Rev. Lett. 86, 1191 (2001).

${ }^{9}$ F. Chandezon, P. M. Hansen, C. Ristori, J. Pedersen, J. Westergaard, and S. Bjornholm, Chem. Phys. Lett. 277, 450 (1997).

${ }^{10}$ J. Borggreen, F. Chandezon, O. Echt, H. Grimley, K. Hansen, P. M. Hansen, and C. Ristori, Eur. Phys. J. D 9, 119 (1999).

${ }^{11}$ W. Branz, Ph.D. thesis, University of Stuttgart, 2001.

${ }^{12}$ W. Branz, N. Malinowski, A. Enders, and T. P. Martin, Phys. Rev. B 66, 092101 (2002).

${ }^{13}$ T. P. Martin, U. Näher, H. Schaber, and U. Zimmermann, Phys. Rev. Lett. 70, 3079 (1993).

${ }^{14}$ T. P. Martin, U. Näher, H. Schaber, and U. Zimmermann, J. Chem. Phys. 100, 2322 (1994).

${ }^{15}$ C. Ellert, M. Schmidt, C. Schmitt, T. Reiners, and H. Haberland, Phys. Rev. Lett. 75, 1731 (1995).

${ }^{16}$ M. Schmidt, Ph.D. thesis, University of Freiburg, 1996.

${ }^{17}$ U. Zimmerman, U. Näher, S. Frank, T. P. Martin, and N. Malinowski, in Large Clusters of Atoms and Molecules, NATO ASI Series E, edited by T. P. Martin (Kluwer Academic, Dordrecht, the Netherlands, 1996), Vol. 313, p. 511.

${ }^{18}$ R. H. Leary and J. P. K. Doye, Phys. Rev. E 60, 6320 (1999).

${ }^{19}$ W. Branz, N. Malinowski, H. Schaber, and T. P. Martin, Chem. Phys. Lett. 328, 245 (2000). 